\title{
Simulation of high Reynolds number flow over a backward facing step using SPH
}

\author{
T. S. Ting* M. Prakash ${ }^{\dagger} \quad$ P. W. Cleary ${ }^{\dagger}$ \\ M. C. Thompson*
}

(Received 10 November 2005; revised 4 August 2006)

\begin{abstract}
The flow over a backward facing step is often used as a test case for analyzing the performance of computational methods and turbulence models. It embodies several important aspects of turbulent flow: flow separation, recirculation, and reattachment. It is convenient that the separation point is fixed at the sharp corner of the step, so no complexities arise from movement of the separation point. We report on high Reynolds number simulations of flow over a backwardfacing step using the Lagrangian Smoothed Particle Hydrodynamics (SPH) method. A preliminary attempt to quantitatively evaluate the ability of SPH to predict high Reynolds number flows is made here.
\end{abstract}

${ }^{*}$ Dept. Mechanical Engineering, Monash University, Melbourne, Australia.

${ }^{\dagger}$ CSIRo Mathematical \& Information Sciences, Melbourne, Australia. mailto:Mahesh.Prakash@csiro.au

See http://anziamj . austms .org.au/V47EMAC2005/Ting for this article, (c) Austral. Mathematical Soc. 2006. Published October 2, 2006. ISSN 1446-8735 
The effect of using a sub-particle scale model within SPH similar in concept to a subgrid scale model in grid-based Large Eddy Simulations is investigated. Simulations were performed at three different SPH resolutions and compared with experimentally and numerically (using traditional grid based CFD) observed velocity profiles and the re-attachment length. The flow Reynolds number was 132,000 based on the inlet velocity and the downstream channel height.

\section{Contents}

1 Introduction

C293

2 SPH modelling

C294

3 LES formulations

C296

4 Wall boundaries

C296

5 Backward facing step

C297

6 Results

C298

7 Discussion

C303

8 Conclusion

C307

References

C307

\section{Introduction}

Smoothed Particle Hydrodynamics (SPH) is a fully Lagrangian computational technique that uses free moving particles to represent a continuum. The 
particles move in response to interactions with surrounding particles sitting within a defined range. SPH has been applied successfully in Computational Fluid Dynamics (CFD) to simulate complex free surface flows, but is rarely used for flows involving separation, reattachment and recirculation. In addition, in most SPH formulations, turbulent effects are neglected.

Formal work in turbulence modelling for SPH is very new. Some of the earliest works were that of Violeau et al. [12] and Monaghan [10]. In this paper, results of SPH simulations of a turbulent flow over a backward-facing step are presented (see Issa et al. [5] for an initial investigation). Here, a Large Eddy Simulation (LES) approach similar to that of Lo \& Shao [8] models the sub-particle (SPS) turbulent scales. The unresolvable stresses that arise from the SPS motion are treated using the Smagorinsky eddy viscosity model. Wall functions are also incorporated to capture the fine scales in the near wall region.

\section{SPH modelling}

Full details of SPH formulations are described by Monaghan [9]. In SPH, the interpolated value of a function $A$ at any position $\mathbf{r}$ is

$$
A(\mathbf{r})=\sum_{b} m_{b} \frac{A_{b}}{\rho_{b}} W\left(\mathbf{r}-\mathbf{r}_{b}, h\right),
$$

where $m_{b}$ and $\rho_{b}$ are the mass and density of particle at $\mathbf{r}$ and the summation is across all particles $b$ within a radius $2 h$ of $\mathbf{r}$. Here $W(\mathbf{r}, h)$ is a $C^{2}$ spline based smoothing kernel that approximates the shape of a Gaussian function but has compact support. Using the idea of interpolants, the Lagrangian Navier-Stokes equations are converted to ordinary differential equations that serve as the equations of motion for the fluid particles. 
The preferred SPH form of the continuity equation is [9]

$$
\frac{d \rho_{a}}{d t}=\sum_{b} m_{b}\left(\mathbf{v}_{a}-\mathbf{v}_{b}\right) \cdot \nabla W_{a b}
$$

where $\rho_{a}$ is particle $a$ 's density; $\mathbf{v}_{a}$ is particle $a$ 's velocity; $m_{b}$ is the mass of neighbour particle $b$, and $W_{a b}=W\left(\mathbf{r}_{a b}, h\right)$. Here, $\mathbf{r}_{a b}=\mathbf{r}_{a}-\mathbf{r}_{b}$ is the position vector of particle $a$ relative to particle $b$. The kernel $W_{a b}$ is evaluated for the particle-particle distance $\left|\mathbf{r}_{a b}\right|$ with smoothing length $h$.

The SPH form of the momentum equation used here is from [1]:

$$
\frac{d \mathbf{v}_{a}}{d t}=\sum_{b} m_{b}\left[\left(\frac{P_{b}}{\rho_{b}^{2}}+\frac{P_{a}}{\rho_{a}^{2}}\right)-\frac{\xi}{\rho_{a} \rho_{b}} \frac{4 \mu_{a} \mu_{b}}{\left(\mu_{a}+\mu_{b}\right)} \frac{\mathbf{v}_{a b} \cdot \mathbf{r}_{a b}}{\mathbf{r}_{a b}^{2}+\eta^{2}}\right] \nabla_{a} W_{a b},
$$

where $P_{a}$ is particle $a$ 's pressure; $\mu_{a}$ is particle $a$ 's viscosity and $\mathbf{v}_{a b}=\mathbf{v}_{a}-\mathbf{v}_{b}$. Here $\xi$ is a factor with a theoretical value of four, and $\eta$ is a small parameter used to smooth out the singularity at $\mathbf{r}_{a b}=0$.

The SPH method used here is a quasi-compressible one. To relate particle density to fluid pressure, the stiff equation of state is

$$
P=P_{0}\left[\left(\frac{\rho}{\rho_{0}}\right)^{\gamma}-1\right]
$$

where $P_{0}$ is the magnitude of the pressure, and $\rho_{0}$ is the reference fluid density. For water the value of $\gamma=7$ is generally used. The pressure scale factor $P_{0}$ is given via

$$
\frac{\gamma P_{0}}{\rho_{0}}=100 V^{2}=c_{s}^{2},
$$

where $V$ is the characteristic or maximum fluid velocity, and $c_{s}$ is the fluid speed of sound. Proper selection of $c_{s}$ ensures that the density variations in the fluid are less than $1 \%$ and the flow thus regarded as incompressible. 


\section{LES formulations}

The core of LES is the filtering procedure described in detail by Wilcox [13]. Filtering of the Navier-Stokes momentum equation results in the sub-particle scale (SPS) stress tensor [8]

$$
\tau_{i j}=\rho\left(\overline{u_{i} u_{j}}-\bar{u}_{i} \bar{u}_{j}\right),
$$

that must be modelled to achieve closure of the equations. Following [8], we used the eddy viscosity approach of Smagorinsky:

$$
\begin{gathered}
\tau_{i j}-\frac{1}{3} \delta_{i j} \tau_{k k}=-2 \mu_{T} \bar{S}_{i j} \\
\mu_{T}=\rho\left(C_{S} \Delta\right)^{2} \sqrt{2 \bar{S}_{i j} \bar{S}_{i j}}
\end{gathered}
$$

where $\bar{S}_{i j}$ is the SPS strain tensor; $C_{S}$ is the Smagorinsky constant which ranges typically from 0.065 to 0.25 and $\Delta$ is the filter width which we define to be the SPH kernel smoothing length, $h$. We use $C_{S}=0.2$ in our work. By using an eddy viscosity assumption for the sps tensor, the filtered momentum equation is then analogous to the Navier-Stokes equations [12]. The eddy viscosity is then incorporated into the SPH momentum equation in a simple manner by using $\left(\mu+\mu_{T}\right)$ instead of $\mu$ in Equation (3).

\section{Wall boundaries}

In SPH, solid walls are also modelled using particles, spaced uniformly according to the initial particle configuration. To avoid inter-penetration of fluid particles, the boundary particles are prescribed with forces - typically of a Lennard-Jones form - that are exerted on the fluid in the normal direction.

In turbulent flow, near wall velocity gradients are difficult to resolve. Here, standard wall functions bridge the gap between the solid walls and the 


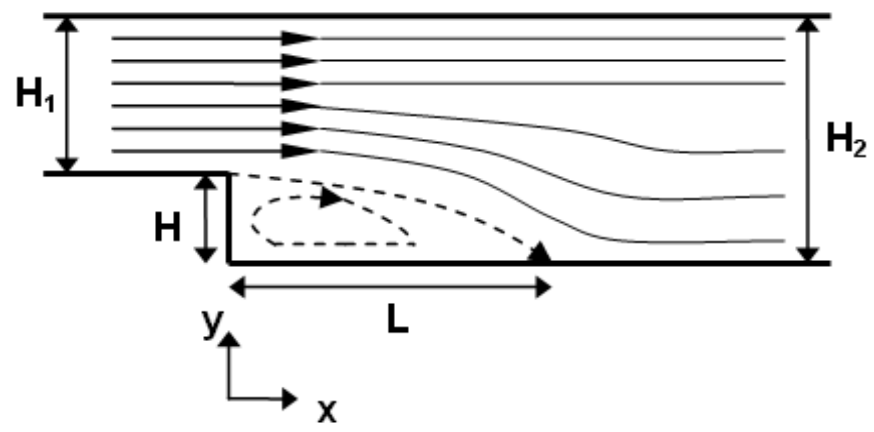

FiguRE 1: Physical geometry and coordinate system.

first layer of particles away from the walls. We chose these wall functions:

$$
\begin{aligned}
& U^{+}=y^{+}, \quad y^{+}<11.6, \\
& U^{+}=2.54 \ln \left(y^{+}\right)+5.56, \quad 11.6<y^{+}<100,
\end{aligned}
$$

as suggested by Houghton \& Carpenter [4]. Here, $U^{+}=\bar{u} / V_{*}$ and $U^{+}=$ $y V_{*} / \nu$, where $V_{*}=\sqrt{\tau_{w} / \rho}$.

The wall shear stress $\tau_{w}$ at each associated boundary particle is calculated using information from particles nearest to the wall. Once $\tau_{w}$ is evaluated across all solid boundaries, $y^{+}$values are assigned to all particles in the nearwall region. The wall functions are then applied on appropriate particles as determined by the conditions in Equations (8) and (9).

\section{$5 \quad$ Backward facing step}

The geometry considered here follows the work of Speziale \& Ngo [11] who based their work on the experimental results of Kim et al. [6]. The expansion ratio is 3:2 $\left(H_{2} / H_{1}\right)$ as shown in Figure 1. A fully developed turbulent channel flow profile is prescribed at the inlet. The Reynolds number is 132,000 
TABLE 1: Mean reattachment points as determined by experiment and numerical computations .

\begin{tabular}{lr}
\hline Case & $L$ \\
\hline Experiment Kim et al. [6] & 7.00 \\
Non-linear $k-\epsilon$ model — Speziale \& Ngo [11] & 6.4 \\
Standard SPH: $N=40062$ & 5.86 \\
Standard SPH: $N=160122$ & 11.24 \\
Standard SPH: $N=360482$ & 16.65 \\
SPH \& Smagorinsky: $N=40062$ & 4.87 \\
SPH \& Smagorinsky: $N=160122$ & 10.00 \\
SPH \& Smagorinsky: $N=360482$ & 13.60 \\
\hline
\end{tabular}

based on the mean inlet centreline velocity $U$ and the downstream channel height $H_{2}$. SPH simulations were performed using $N=40062,160122$ and 360482 particles.

\section{Results}

The results of the SPH simulations are primarily compared with the experimental results of Kim et al. [6]. Kim et al. [6] measured the mean reattachment length $(L=x / H)$ to be 7.0. Speziale \& Ngo [11] were able to obtain best results of 6.4 using a nonlinear $k$ - $\epsilon$ model.

Figure 2 shows the mean axial velocity $(\bar{u} / U)$ profiles at specific streamwise locations behind the step as computed using standard SPH, plotted together with the experimental results of Kim et al. [6]. Figure 3 shows the streamlines generated from the standard SPH and $k-\epsilon$ model computations of Speziale \& Ngo [11]. The SPH results are generated using a time averaged solution of the statistically steady flow field. The state of the flow was monitored by capturing time histories of $x$-velocity at different locations in 
(a) $x / H=1.333$

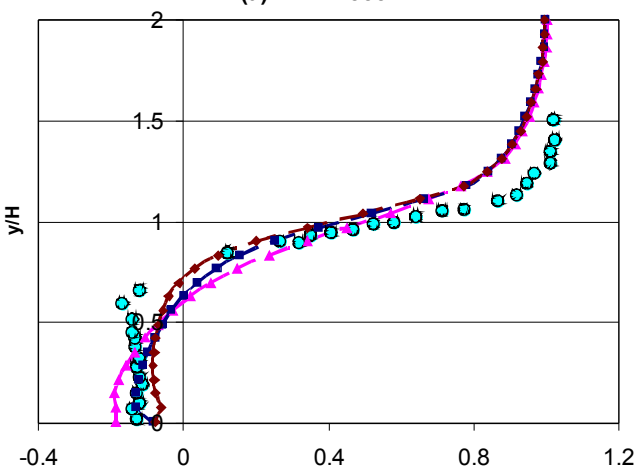

(c) $x / H=6.220$

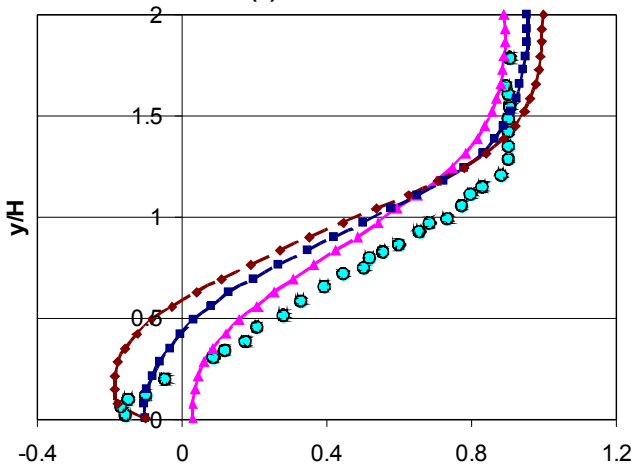

(b) $\mathrm{x} / \mathrm{H}=2.667$

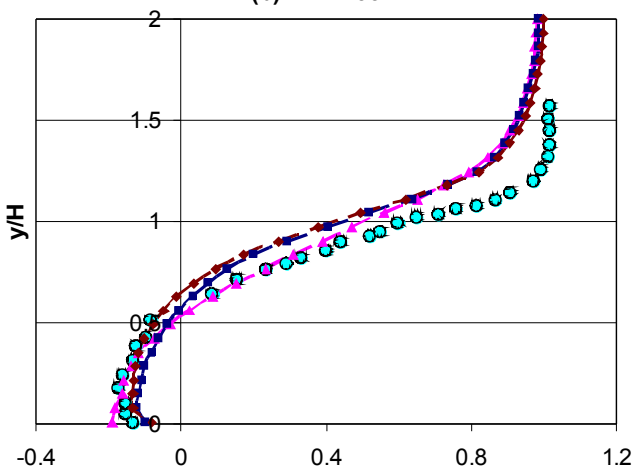

(d) $\mathrm{x} / \mathrm{H}=8.000$

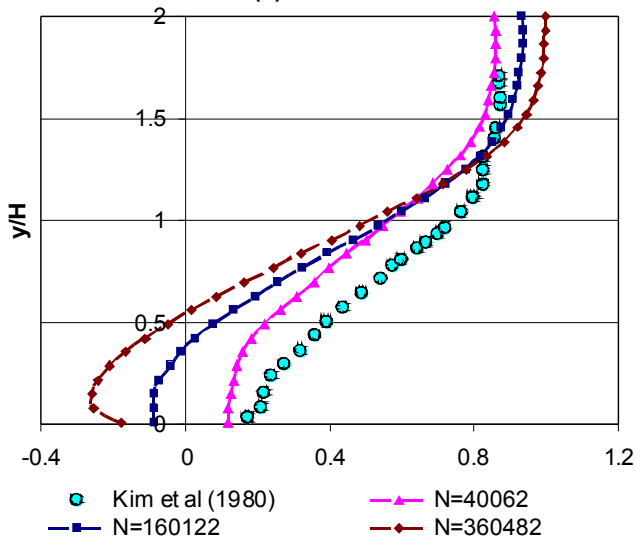

Figure 2: Profiles of mean axial velocity, $\bar{u} / U$, at selected streamwise locations. Computations using standard SPH. 
(a)

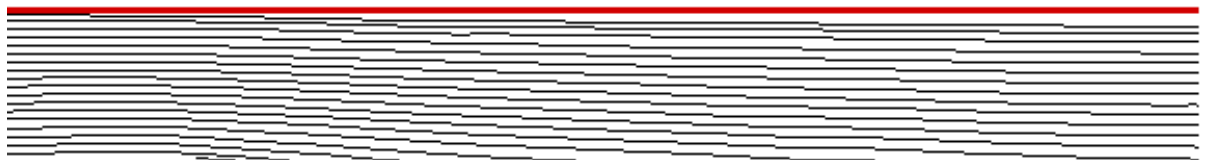

(b)

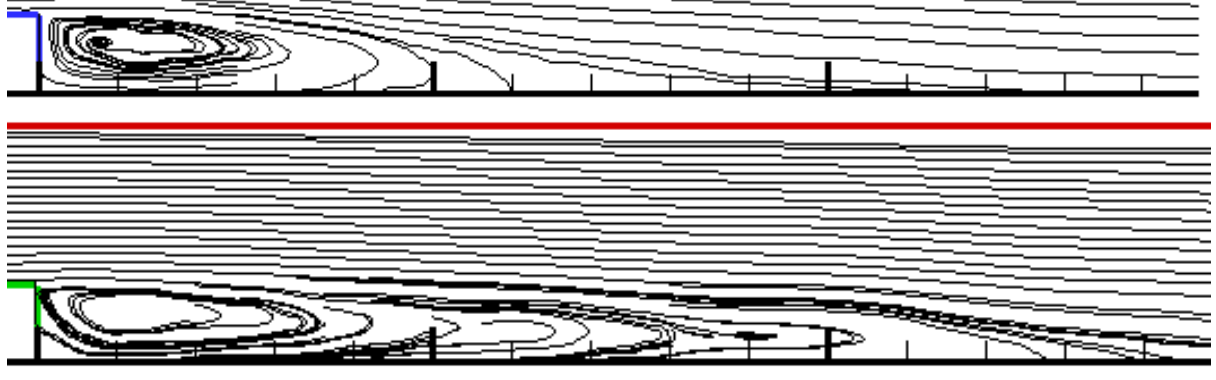

(c)

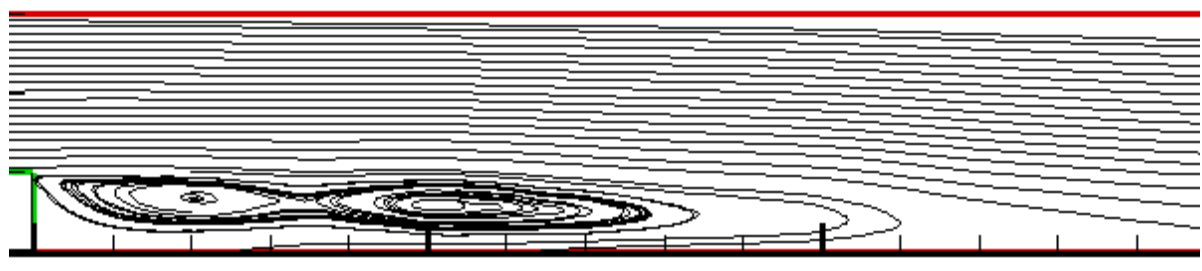

(d)

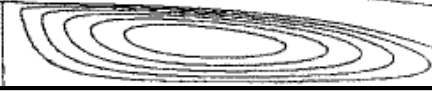

FigurE 3: Comparison of generated streamlines using standard SPH (timeaveraged over several seconds), (a) $N=40062$, (b) $N=160122$, (c) $N=$ 360482 , (d) Speziale \& Ngo [11], nonlinear $k$ - $\epsilon$ model. 
(a) $x / H=1.333$

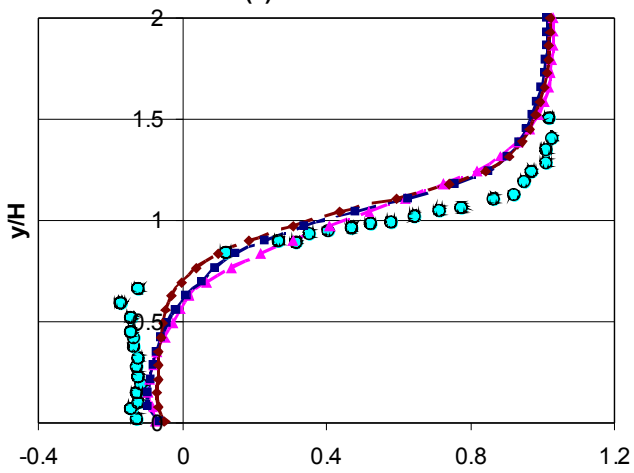

(c) $x / H=6.220$

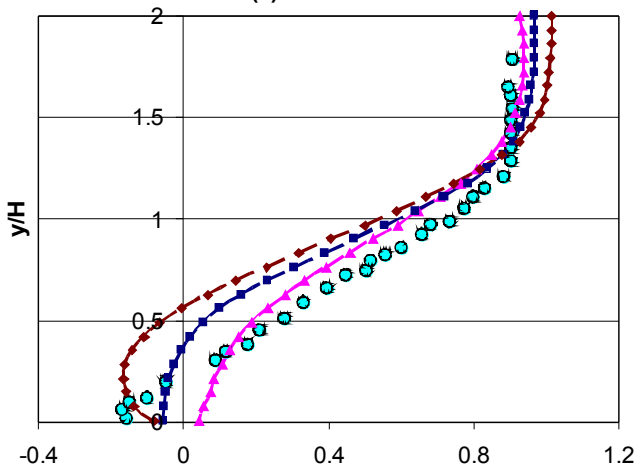

(b) $\mathrm{x} / \mathrm{H}=\mathbf{2 . 6 6 7}$

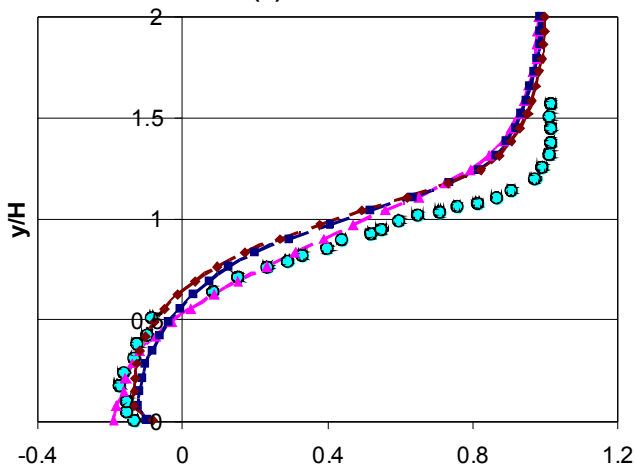

(d) $\mathrm{x} / \mathrm{H}=8.000$

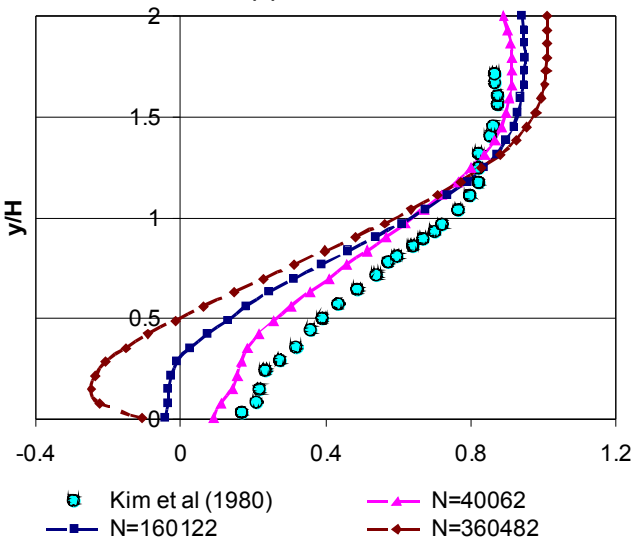

Figure 4: Profiles of mean axial velocity, $\bar{u} / U$, at selected streamwise locations. Computations using standard SPH with Smagorinsky model. 
(a)

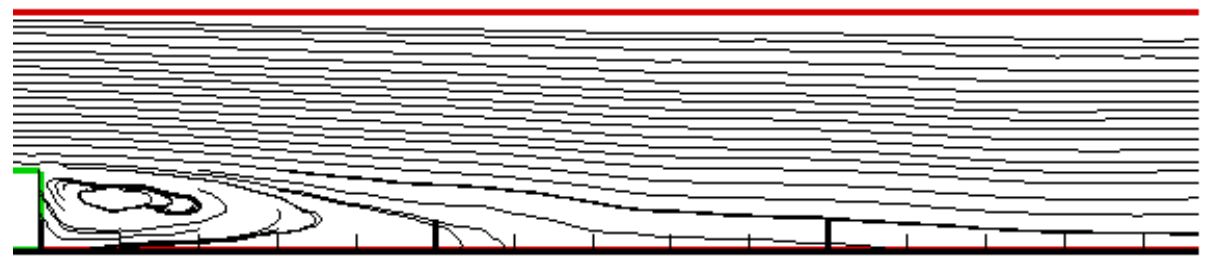

(b)

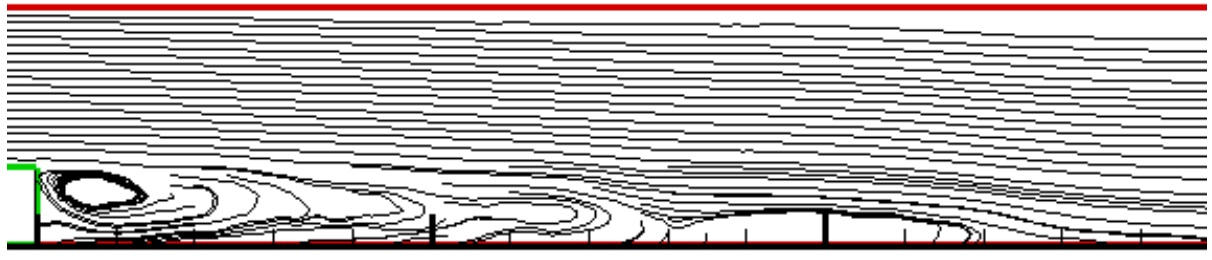

(c)

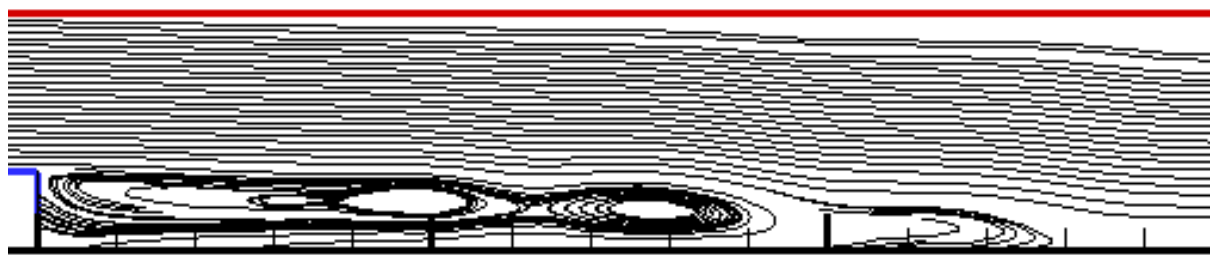

Figure 5: Comparison of generated streamlines using standard SPH \& Smagorinsky (time-averaged over several seconds), (a) $N=40062$, (b) $N=$ 160122 , (c) $N=360482$. 
the recirculation zone. Once the time histories show a statistically steady behaviour, time averaging was performed over several seconds of simulation time.

Examination of Figure 2 shows that computations using $N=40062$ particles offered results that compare best with that from the experiment. At a higher resolution, $N=160122$, standard SPH predicted a much longer recirculation zone behind the step. At the highest resolution $N=360482$, two recirculation bubbles are predicted by SPH (Figure 3(c)); one centred at approximately $x / H=2$, and the second at $x / H=6$. The mean reattachment location of the free shear layer emanating from the step is determined by the location of zero $x$-velocity (along the lower wall behind the step) of the time averaged flow field. Table 1 shows the calculated mean reattachment lengths for the three cases.

Figure 4 shows the mean velocity profiles as computed after implementing the Smagorinsky model and wall functions. Streamlines of the corresponding time-averaged flow fields are shown in Figure 5.

The mean reattachment lengths computed after incorporating the models are also tabulated in Table 1. Comparison of reattachment lengths and close examination of Figure 5 shows that use of the Smagorinsky model did not significantly improve the SPH predictions. Very large mean recirculation zones were still being computed at the higher resolutions. The mean velocity profiles also do not appear to converge on a particular result even though increasing number of particles were being used.

\section{Discussion}

The effect of using the Smagorinsky model is to dissipate the fluctuations in a turbulent flow. For the flow being considered here, the dissipation is evident in the region close to the walls, where on average the particles are moving 

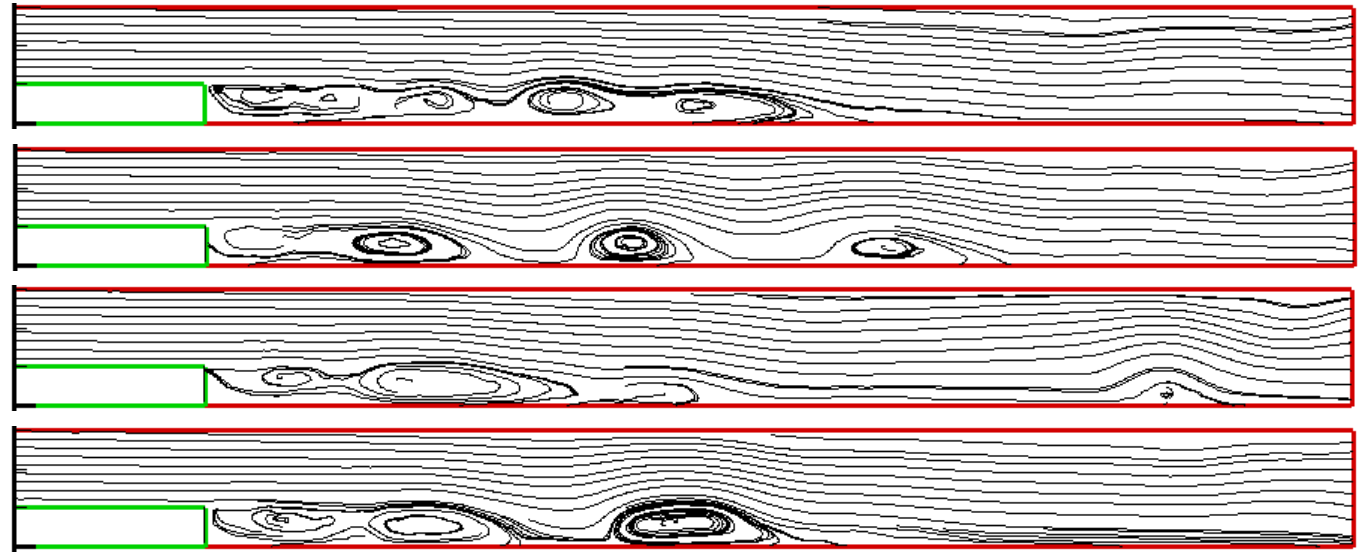

FiguRE 6: Evolution of the SPH flow field after approximately 30 seconds of simulation time, snapshots taken at 8 second intervals.

slower. Closer examination of the numerical values indicate that velocity gradients in the near wall region tended to be more pronounced after the turbulence models were incorporated. Different numerical procedures for the wall functions may alter these findings; in this work, a single iteration using the instantaneous wall shear stress $\tau_{w}$ was used. Alternatives include using $\tau_{w}$ from the previous time-step, or to use multiple iterations with the current procedure.

The elongation of the recirculation bubbles as predicted for cases $N=$ 160122 and $N=360482$ differ markedly from the computations using the nonlinear $k-\epsilon$ model (see Figure 3(b)-(c) or 5(b)-(c)). At a Reynolds number of 132000 it is reasonable to expect that the flow should be fully turbulent and hence strongly variable in time. It is possible that with more particles, certain time varying features in the flow that were not seen in simulations at lower resolution were being captured. To investigate this, the evolution of the flow past the backward facing step is examined for the case $N=360482$. In the SPH simulations that were performed, the flow was observed to tend to a statistically steady behaviour after 30 seconds of simulation time. Figure 6 
depicts the flow evolution after this time has been reached.

As Figure 6 clearly shows, the flow field is very unsteady even after the transient phase. Fluctuation of the primary recirculation zone is observed. Le et al. [7] performed a very comprehensive study of the backward-facing step flow using 3D Direct Numerical Solution (DNS), and also reported fluctuations of the reattachment length in the range of $x / H=5$ to 8 for their geometry.

Figure 6 also shows two other distinct flow characteristics: the formation and detachment of secondary and tertiary recirculation zones behind the reattachment, and the outside presence of recirculation regions on the upper wall. Both characteristics are rarely investigated by researchers, but Le et al. [7] did report the presence of spanwise vortices situated behind the reattachment point. Our findings are further confirmed by comparison with the work of the FEAST Group [3]. They performed a 2D simulation of a high Reynolds number backward facing step flow (in the range of $R e=10^{4}$ ) using a finite element approach, and are shown here in Figure 7. Figure 7 shows very clearly flow characteristics that are similar to those found in Figure 6, mainly the existence of downstream recirculation zones past the reattachment point. Recirculation zones on the upper wall and fluctuations of the primary recirculation zone are also clearly depicted.

Although our observations of the backward -facing step flow evolution may compare qualitatively with the findings of other researchers, some discrepancies have been found in the quantitative values especially the mean reattachment point. We postulate that the numerical discrepancies are primarily due to the limitations of a $2 \mathrm{D}$ computation. As far as fully turbulent flows are concerned, a LES computation for such a flow should be performed in $3 \mathrm{D}$ for best results. In a 2D computation, the vortex stretching mechanism in the spanwise direction is lost, and the velocity scales will not be resolved correctly. Subsequently, the energies in the turbulent spectra will not be distributed properly, which may cause an accumulation of energy at the incorrect scales. This is one of the possible causes for the large fluctuations 

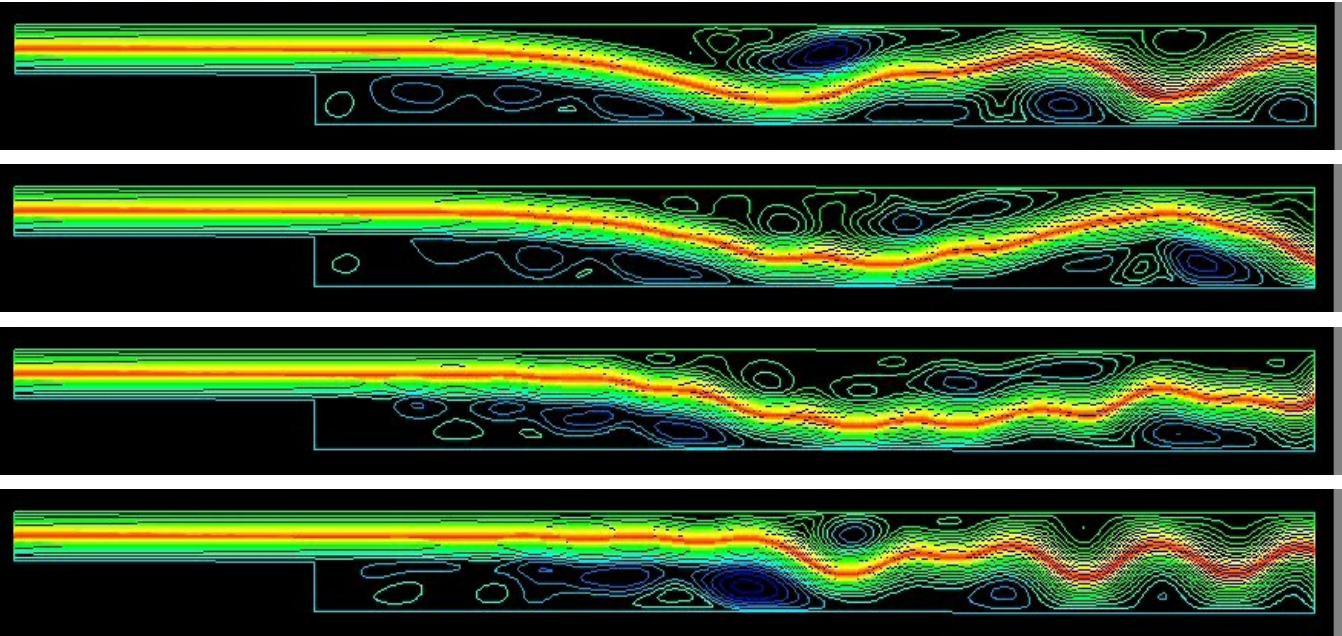

FigURE 7: 2D visualisation of turbulent flow past backward-facing step with $R e \approx 10^{4}$. Streamlines computed by the FEAst Group [3]. Snapshots taken at four second intervals

observed in our work.

A further possibility could be the methodology used to time average the SPH solution. In the present work we have used the same time sample for averaging simulations at all three resolutions. At higher resolutions we note that SPH is capturing flow structures that are time varying in nature; depending on their periodicity, the time averaging procedure would probably demand longer sampling windows. This aspect of the averaging process will be the subject of analysis for the future.

However, the baseline SPH code performed fairly well in the prescribed flow conditions even without the presence of any turbulence modelling. Cleary \& Monaghan [2] suggested that dissipative motions at the sub-particle scale exist in SPH, which prevents the excessive accumulation of energy in the larger scales. Therefore, it is possible to think of SPH as a natural LES technique, with an inherent sub-particle scale model. 


\section{Conclusion}

A quasi-compressible SPH method incorporated with the Smagorinsky eddy viscosity model is used to simulate a high Reynolds number time dependent flow past a backward facing step. The mean flow statistics and streamlines were computed, and compared with experimental results and a numerical solution at steady state using a commercial grid based package. The SPH code performed fairly well in the prescribed flow conditions even without the presence of any turbulence modelling. Some deviations between SPH and the experiment were found at downstream locations from the step.

At fine resolutions, mean streamlines generated using the SPH flow field showed multiple recirculation zones which were not seen in the steady state grid based solution. Further investigation revealed that such patterns have been reported where researchers used time dependent finite element solutions.

We conclude that deviations in the numerical values when compared with experiment are primarily due to the limitations of a $2 \mathrm{D}$ computation. This will need to be confirmed through a comprehensive analysis of a 3D computation as part of ongoing work. The sampling procedure of the unsteady flow field needed in time averaging the SPH solutions may require examination for computations at higher resolutions.

\section{References}

[1] Cleary, P. W., Modelling confined multi-material and heat mass flows using SPH, Applied Mathematical Modelling, 22, 981-993, 1998. C295

[2] Cleary, P. W. and Monaghan, J. J., Boundary interactions and transition to turbulence for standard CFD problems using SPH, Proceedings of the 6th Biennial Conference on Computational 
Techniques and Applications, Canberra, ACT, 1993, pages 157-165, World Scientific, 1994. C306

[3] FEAST Group, Flow over a backward facing step at high Reynolds number. [Online] http://www.featflow.de/album/catalog/bf s_ high_2d/data.html [Accessed26/09/2005]. C305, C306

[4] Houghton, E. L. and Carpenter, P. W., Aerodynamics for Engineering Students, 5th edition, Butterworth-Heinemann, Burlington, MA, 2003. C297

[5] Issa, R., Lee, E. S. and Violeau, D., Incompressible separated flows simulations with the smoothed particle hydrodynamics gridless method, Int. J. Numer. Meth. Fluids, 47, 1101-1106, 2005. C294

[6] Kim, J., Kline, S. J. and Johnston, J. P., ASME J. Fluids Enging, 102, 302, 1980. C297, C298

[7] Le, H., Moin, P. and Kim, J., Direct numerical simulation of turbulent flow over a backward-facing step, J. Fluid Mech., 330, 349-374, 1997. C305

[8] Lo, E. Y. M. and Shao, S., Simulation of near-shore solitary wave mechanics by an incompressible SPH method, Applied Ocean Research, 24, 275-286, 2002. C294, C296

[9] Monaghan, J. J., Smoothed particle hydrodynamics, Ann. Rev. Astron. Astrophys., 30, 543-574, 1992. C294, C295

[10] Monaghan, J. J., SPH compressible turbulence, Monthly Notices of the Royal Astronomical Society, 335, 843-852, 2002. C294

[11] Speziale, C. G. and Ngo, T., Numerical solution of turbulent flow past a backward-facing step using a nonlinear $k-\epsilon$ model, Int. J. Engng. Sci., 10, 1099-1112, 1988. C297, C298, C300 
[12] Violeau, D., Piccon, S. and Chabard, J-P., Two attempts of turbulence modelling in smoothed particle hydrodynamics, Proceedings of the 8th International Symposium on Flow Modelling and Turbulence Measurement, Tokyo, Japan, 4-6 December 2001, World Scientific, 2002. C294, C296

[13] Wilcox, D. C., Turbulence Modelling For CFD, DCW Industries, Inc., La Canada, California, 1993. C296 